\title{
Reduced prefrontal gyrification in obsessive-compulsive disorder
}

\author{
Thomas Wobrock • Oliver Gruber • Andrew M. McIntosh • Susanne Kraft • \\ Anne Klinghardt · Harald Scherk • Wolfgang Reith • Thomas Schneider-Axmann • \\ Stephen M. Lawrie · Peter Falkai · Thomas William Moorhead
}

Received: 4 September 2009/Accepted: 23 December 2009/Published online: 29 January 2010

(C) The Author(s) 2010. This article is published with open access at Springerlink.com

\begin{abstract}
Structural magnetic resonance imaging (MRI) studies reveal evidence for brain abnormalities in obsessive-compulsive disorder (OCD), for instance, reduction of gray matter volume in the prefrontal cortex. Disturbances of gyrification in the prefrontal cortex have been described several times in schizophrenia pointing to a neurodevelopmental etiology, while gyrification has not been studied so far in OCD patients. In 26 OCD patients and 38 healthy control subjects MR-imaging was performed. Prefrontal cortical folding (gyrification) was measured bilaterally by an automated version of the automated-gyrification index (A-GI), a ratio reflecting the extent of folding, from the slice containing the inner genu of the corpus callosum up to the frontal pole. Analysis of covariance (ANCOVA, independent factor diagnosis, covariates age, duration of education) demonstrated that compared with control subjects, patients with OCD displayed a significantly reduced A-GI in the left hemisphere $(p=0.021)$ and a trend for a decreased A-GI in the right hemisphere $(p=0.076)$.
\end{abstract}

T. Wobrock $(\bowtie) \cdot$ O. Gruber · A. Klinghardt · H. Scherk ·

T. Schneider-Axmann - P. Falkai

Department of Psychiatry and Psychotherapy,

Georg-August-University, Von-Siebold-Str. 5,

37075 Göttingen, Germany

e-mail: twobroc@gwdg.de

A. M. McIntosh - S. M. Lawrie - T. W. Moorhead Department of Psychiatry, University of Edinburgh, Edinburgh, UK

\section{S. Kraft}

Department of Psychiatry and Psychotherapy,

University of the Saarland, Homburg/Saar, Germany

W. Reith

Department of Neuroradiology,

University of the Saarland, Homburg/Saar, Germany
Significant correlations between prefrontal lobe volume and A-GI were only observed in controls, but not in OCD patients. In conclusion, prefrontal hypogyrification in OCD patients may be a structural correlate of the impairment in executive function of this patient group and may point to a neurodevelopmental origin of this disease.

Keywords Obsessive-compulsive disorder (OCD) . Magnetic resonance imaging (MRI) - Gyrification . Cortical folding $\cdot$ Hypogyria

\section{Introduction}

About 1-3\% of the worlds' population suffer from obsessive-compulsive disorder (OCD) [4, 20], which causes a high individual and socioeconomic burden [21]. Clinically, OCD is characterized by intrusive unwanted thoughts, ideas, or images that are distressing (obsessions) and urges to perform ritualistic behaviors or mental acts (compulsions) to reduce this distress. Although symptoms tend to wax and wane through the course of the disorder, OCD symptoms rarely remit spontaneously.

Brain imaging techniques suggest that specific neuronal circuits are responsible for the onset and mediation of OCD symptoms [38]. Several structural magnetic resonance imaging (MRI) studies revealed evidence for the presence of brain abnormalities in $\mathrm{OCD}$, mainly observing reduced volumes in prefrontal, cingulate, thalamic, and temporolimbic regions [e.g. 30, 40]. In a meta-analysis of brain volume changes in obsessive-compulsive disorder no volumetric differences were found for the whole brain, intracranial region, gray matter, or prefrontal cortex, but OCD patients did show a reduced volume of the left anterior cingulate cortex and the left and right orbitofrontal 
cortex compared with control subjects [34]. Functional imaging studies reported metabolic and functional abnormalities in nearly the same brain regions, predominantly involving not only the orbitofrontal and anterior cingulate cortices, basal ganglia, and thalamus [e.g. 24, 26, 39, 41], but also lateral prefrontal cortices [16]. With regard to these findings, a dysfunction of particularly prefrontalstriatal-thalamic-circuitry has been implicated in the pathophysiology of OCD [41].

In schizophrenia as well as in OCD, changes in shape complexity measured by fractal dimension of cerebral cortical surface were observed when comparing to healthy individuals [12]. Both conditions are associated with an increased heritability, and both disorders are assumed of at least in part neurodevelopmental origin [33, 45]. Disturbances of cortical folding are thought to be related to abnormal cerebral development, particularly to anomalies of cortico-cortical [42] and possibly subcortico-cortical connectivity [31]. Cortical folding of the human brain can be determined using the gyrification index (GI), which is a ratio of the inner and the outer contours of the brain [48]. The GI increases proportionally to the number of gyri [13]. During prenatal brain development the GI increases approximately up to the age of one, declines to a stable adult value, and seems to be independent of age-associated atrophy [2].

In schizophrenia, abnormalities of cortical folding compared with healthy controls, especially of frontal and temporal regions, have been demonstrated in several studies through MR imaging and post mortem [13, 43, 44]. In a family study, frontal hypergyrification was confirmed in family members suffering from schizophrenia and in unaffected first-degree relatives compared with healthy control subjects [8] and in high-risk individuals for the development of schizophrenia increased right prefrontal lobe GI served as a predictor for the subsequent progression into schizophrenic disease [14].

While there is enough evidence for disturbed function and abnormal structure, especially of prefrontal cortex in OCD, to our knowledge, no studies were carried out to examine cortical folding as a potential biological marker for the latter disease. In the present study, we used an automated, well-validated method for determining the automated-gyrification index (A-GI) for the comparison of prefrontal cortical folding in OCD patients versus healthy control subjects [27].

\section{Methods}

\section{Subjects}

Participants of the study were recruited from the Department of Psychiatry and Psychotherapy, Saarland
University, between 2003 and 2007. Diagnostically, the sample consisted of 26 patients with OCD (DSM-IV criteria) and 38 healthy control subjects. Subjects suffering from dementia, neurological illnesses, severe brain injuries, or brain tumors at the time of examination were excluded from the sample. Subjects having a first-degree relative with schizophrenia spectrum disorder were also excluded. Demographic details by diagnostic group are given in Table 1.

After a complete description of the study, written informed consent was obtained from each patient. The local ethics committees approved the protocol, which is in accordance with the Declaration of Helsinki.

\section{Diagnostic procedure}

The following standardized examinations were performed on each subject: a detailed biographic interview and a consensus diagnosis based on SCID I and II interviews of two independent psychiatrists. The status of healthy controls was confirmed using SCID I and II. For the OCD patients, duration of illness, age of onset, depressive symptoms with the beck depression inventory (BDI) [5], and severity of OCD symptoms using the Yale-Brown Obsessive Compulsive Scale (Y-BOCS) [10] were assessed.

\section{Neuropsychological assessment}

To assess cognitive performance of frontal lobe function, we applied a small test battery focusing on executive function. For the detection of deficits in concept formation, cognitive flexibility, and executive functions, the Wisconsin Card Sorting Test (WCST [15]), and the Tower of London test (TOL; [37]) were used. Additionally, a test tapping the controlled integration of information from different sensory channels, that is, intermodal comparison (IC, "Intermodaler Vergleich", subtest of the Attention Test Battery, TAP, Zimmermann, Fimm/PSYTEST) was conducted. For assessment of psychomotor performance and speed, we used the "Zahlenverbindungstest" (ZVT; [28]), a test similar to the trail-making test (TMT, part A).

\section{MRI acquisition}

MRI scanning was performed on a $1.5 \mathrm{~T}$ Siemens-Magnetom using a T1-weighted MPRAGE sequence with 176 consecutive slices of $1 \mathrm{~mm}$ thickness and a pixel size of $1 \times 1 \mathrm{~mm}^{2}$. Prior to analysis, scans were realigned so that the coronal plane was orthogonal to the AC-PC line.

Details on image preprocessing operations were described in previous work [27]. Briefly summarized, the SPM package (http://www.fil.ion.ucl.ac.uk/spm/) [3] was used to 


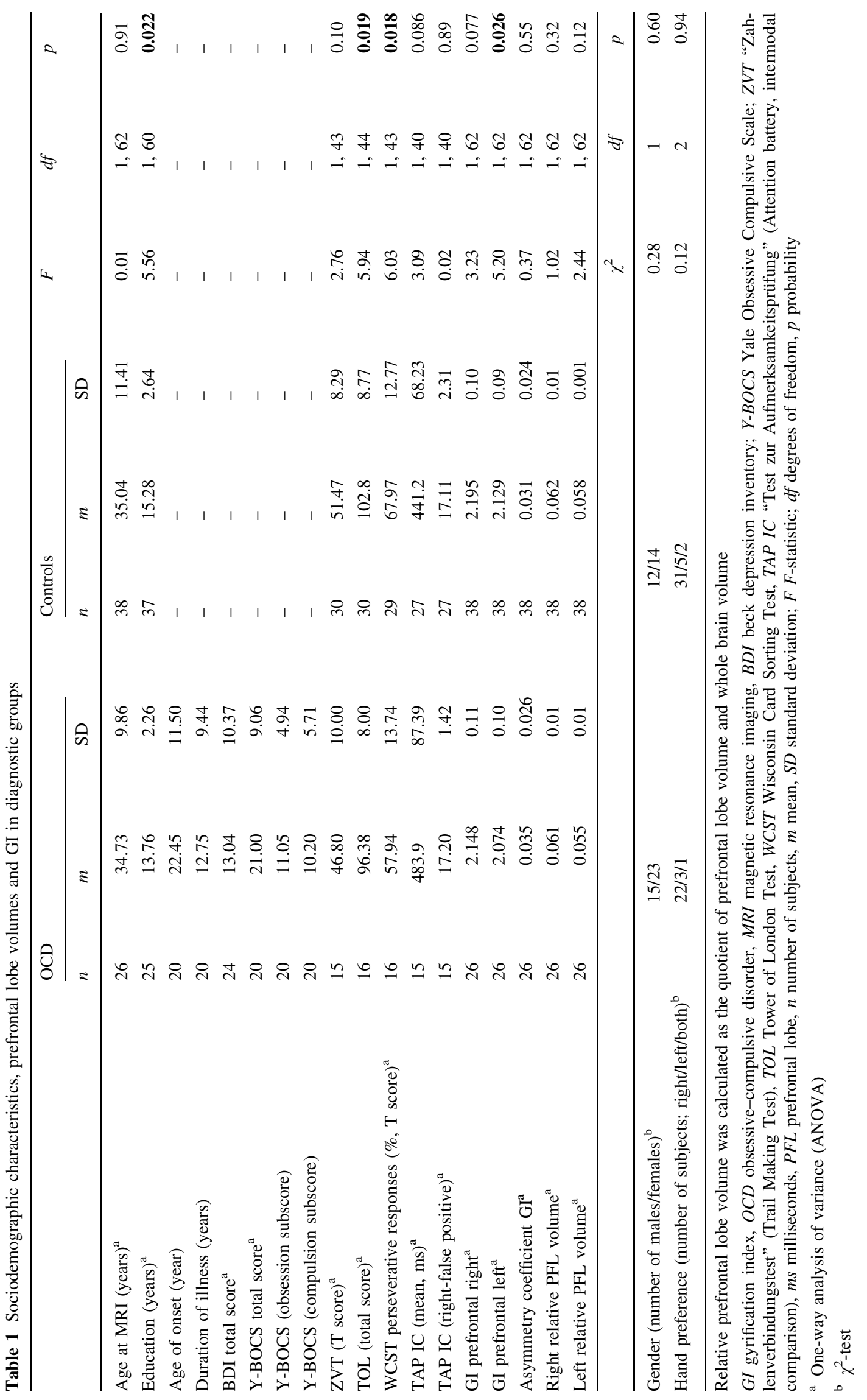


perform preprocessing functions on images used in this study. The T1 candidate scans were segmented in native space using the SPM segment function, after which the SPM brain extraction function recovered a tissue mask for each scan. These masks were combined with the native T1s to give each brain a T1 tissue image with nonbrain tissue and CSF removed. The SPM coregistration function provided a mapping of the extracted brains into the Montreal Neurological Institute (MNI) space to obtain AC-PC registration while maintaining the native space volumes. This mapping into the MNI space without size or shape adjustment was applied to the original $\mathrm{T} 1$, the brain tissue mask, and the three native space segments. These AC-PC registered images were then resliced to $1 \times 1 \times 1 \mathrm{~cm}^{2}$ voxel size, and they formed the base data structure, upon which automated-gyrification index (A-GI) was implemented. Volumes of total gray matter (GM), total white matter (WM), and cerebrospinal fluid (CSF) were obtained as per SPM voxel-based morphometry (VBM) protocols using a MATLAB algorithm [3]. Volumes of the prefrontal lobes were obtained using an automated parcellation procedure implemented in the A-GI software [6, 32], and relative prefrontal volumes were calculated by dividing the absolute prefrontal lobe volumes through whole brain volume (sum of GM, WM and CSF).

\section{Automated-gyrification index (A-GI) processing}

A full description of the A-GI process was given elsewhere $[6,27]$. Briefly, the process can be divided into two procedures, the prefrontal lobe detection and the A-GI extraction. In the first procedure, an automated parcellation procedure is used to locate the left and right prefrontal lobes. In the second automated procedure, the inner and outer contours are traced. In A-GI, the inner trace is a composite of the exposed surface and closed sulci tracings. The outer contour overlaps the inner contour exposed surface traces, except where open sulci occur near the cortical surface. Once the outer and inner contours have been traced, the GI values are calculated for each slice and exported to a tab-delimited text file format. Measurements were taken on every $1 \mathrm{~mm}$ prefrontal slice, approximating 40 slices per brain. The GI values for each slice within the region were summed and then divided by the number of slices, producing a mean GI value for each individual. In Fig. 1a and b, the outer and inner A-GI contour of one prefrontal slice is illustrated.

\section{Statistical analysis}

The A-GI tool is deterministic and its repeated application to the same scan will generate an intraclass correlation co-efficient (ICC) value of 1.00. In a same-subject rescan
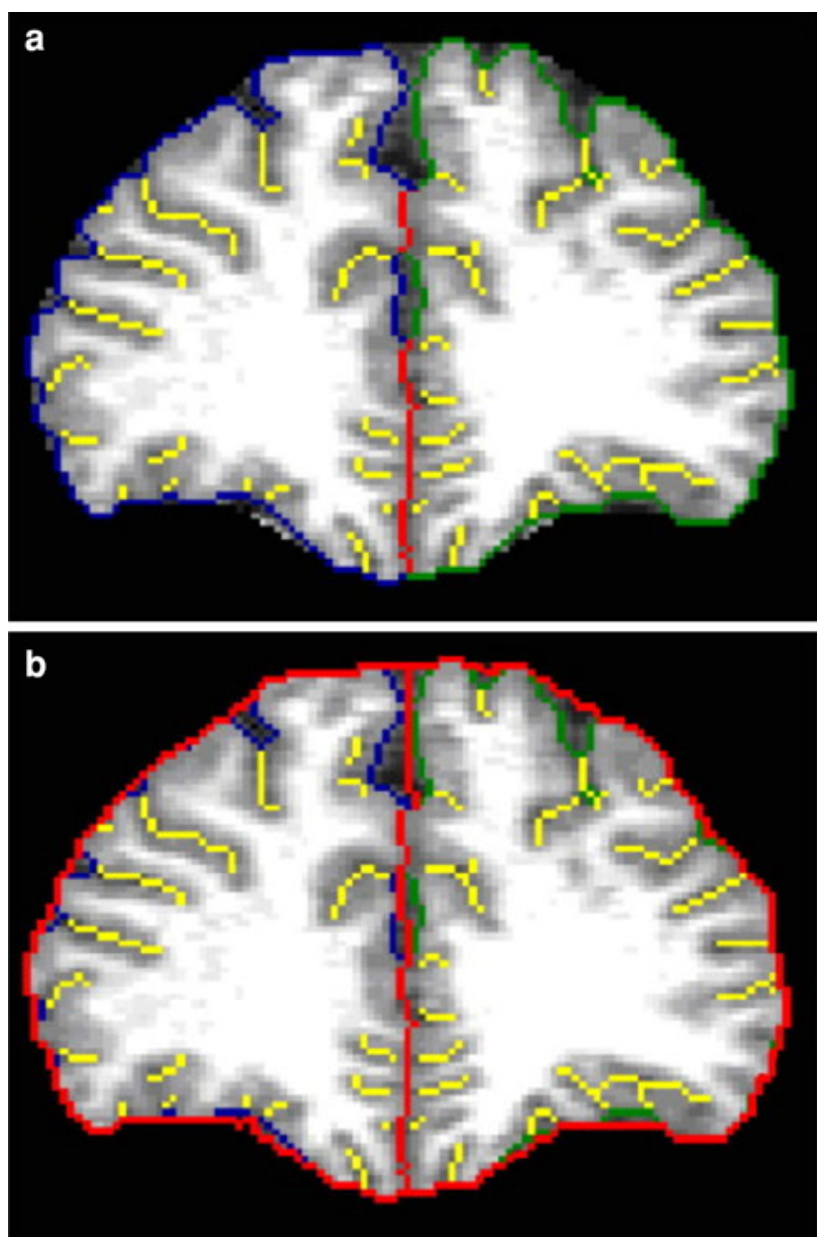

Fig. 1 a Automated GI-inner contour legend: A-GI total inner contour trace on a coronal slice of a control subject. Colors: blue exposed inner contour right; green exposed inner contour left: yellow buried inners. A-GI automated gyrification index. b Automated GIouter contour legend: A-GI total outer contour trace on a coronal slice of a control subject; inner contours overlaid with smoothed outer and IFH trace in red. A-GI automated gyrification index; IFH interhemisphere fissure

repeatability test, it was demonstrated that the A-GI tool obtained an ICC of 0.99 , reflecting a low susceptibility to scanner noise [27]. Equivalent discriminatory power and repeated findings were also demonstrated in the comparison between manual and A-GI [27].

All statistical testing was conducted with SPSS for Windows 14 (SPSS Inc., Chicago, IL, USA). All tests were two-tailed. The level of significance was set at $\alpha=0.05$. Dependent variables were right and left A-GI, and their asymmetry coefficient $\left(2 \times\left(\mathrm{A}-\mathrm{GI}_{\text {right }}-\mathrm{A}-\mathrm{GI}_{\text {left }}\right) /\right.$ $\left(\mathrm{A}-\mathrm{GI}_{\text {right }}+\mathrm{A}-\mathrm{GI}_{\text {left }}\right)$, and in an additional analysis relative prefrontal lobe volume. Independent variable was diagnosis (OCD patients, healthy controls). For dependent variables, the normality assumption was inspected with the Kolmogorov-Smirnov test. 
Oneway analysis of variance (ANOVA) was performed to analyze if there were significant age or education differences between the two diagnostic groups. Chi-square test on independence was used to analyze if the distribution of gender and hand preference was different between the groups. Pearsons product-moment-correlations between dependent variables and age as well as duration of education were calculated for both diagnostic groups. For the control sample, dependent variables were inspected on gender and hand preference differences with oneway ANOVA.

These preliminary statistics determined the main analysis. Since age and education correlated significantly with A-GI values, analysis of covariance (ANCOVA) with independent factor diagnosis and covariates age and duration of education was performed.

To investigate the influence of clinical variables, partial correlations adjusted for age and duration of education between A-GI and duration of illness, age of onset of the disease, Y-BOCS, and BDI were computed. To determine the influence of brain volume on A-GI, partial correlations (PC) adjusted for age between relative prefrontal lobe volume and corresponding A-GI were calculated.

\section{Results}

Sociodemographic, clinical, and neurocognitive parameters

The patients suffering from OCD were significantly less educated than the healthy control subjects; there were no significant differences between the groups with regard to gender, age, and handedness. The cognitive performance of OCD patients compared with control subjects focussing on executive functions was significantly reduced in the WCST (persverative reponses; $p=0.018$ ) and TOL (total score; $p=0.019)$. In addition, OCD patients showed a prolonged reaction time in the intermodal comparison subtest of the TAP at a trend level. Detailed sociodemographic, clinical, and neuropsychological data as well as GI measurements of prefrontal lobe are presented in Table 1.

\section{Gyrification index (GI)}

For all dependent variables there were no significant deviations from the normality assumption. There was a significant negative correlation between age and A-GI on both sides and in both diagnostic groups $(p<0.05$ ) (for details see Fig. 2). In addition, left and right A-GI were significantly positive correlated with duration of education in the total group of subjects $(r=0.34, p=0.01$ for right A-GI and $r=0.36, p<0.001$ for left A-GI) and in the subgroup of control subjects $(r=0.36, p=0.03$; both sides), but not in OCD patients.

Analysis of covariance (ANCOVA; factor diagnosis, intervening variables age and duration of education) demonstrated that compared with control subjects, patients with OCD displayed a significantly reduced A-GI on the left side $(d f=1,58 ; F=5.65 ; p=0.021)$ and a trend for a decreased A-GI on the right side $(d f=1,58 ; F=3.26$; $p=0.076$ ). No significant difference was found with regard to the asymmetry coefficient of the GI (for details see Table 2).

There were no significant differences in GI between males and females in our study sample. However, to rule out a significant influence of gender on dependent variables, we calculated an additional ANOVA (diagnostic group $\times$ gender) showing no significant effect of gender.

There were no significant correlations between A-GI values and duration of illness, age of onset of the disease, Y-BOCS (total score), or BDI (total score). In addition, we
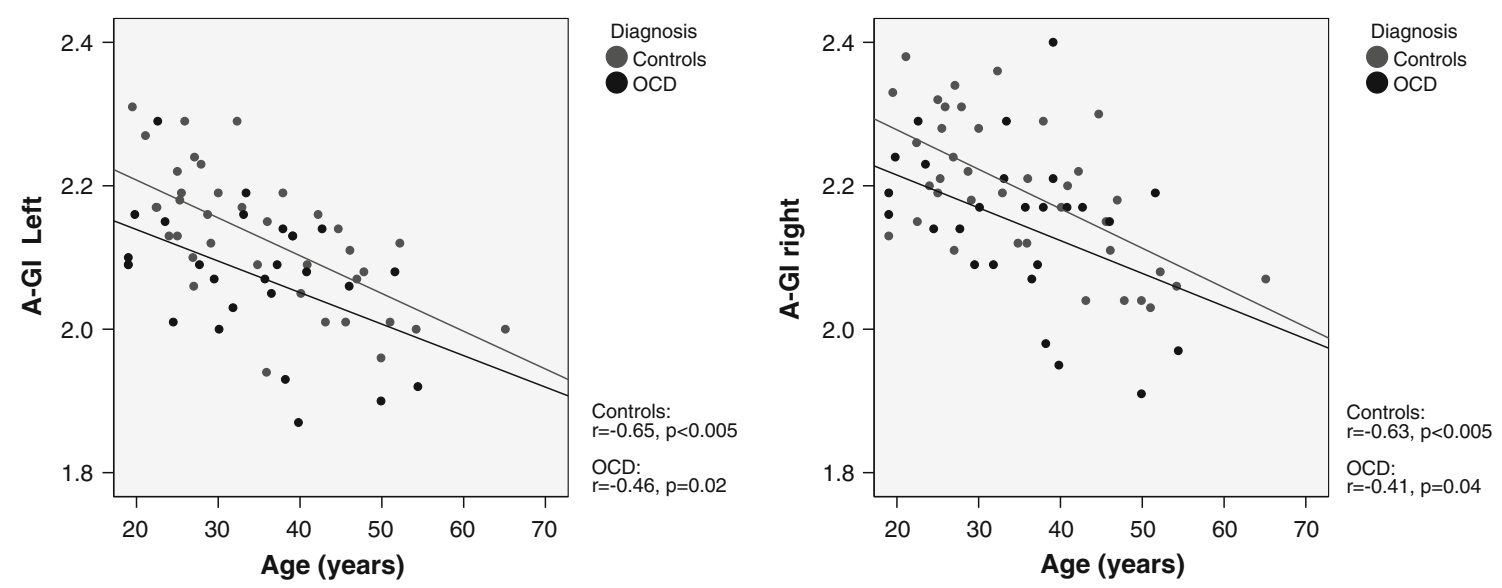

Fig. 2 Relationship between prefrontal GI and age legend: correlation between left and right prefrontal GI and age. GI gyrification index 
Table 2 Gyrification and prefrontal lobe volume in OCD versus control subjects

\begin{tabular}{lllllll}
\hline & Age & \multicolumn{2}{l}{ Education } & \multicolumn{4}{l}{ Diagnosis } \\
\cline { 5 - 7 } & $p$ & $p$ & $F$ & $d f$ & $p$ \\
\hline GI right $^{\mathrm{a}}$ & $<0.0005$ & 0.23 & 3.3 & 1,58 & 0.076 \\
GI left $^{\mathrm{a}}$ & $<0.0005$ & 0.20 & 5.7 & 1,58 & $\mathbf{0 . 0 2 1}$ \\
GI asymmetry coefficient $^{\mathrm{b}}$ & - & - & 0.3 & 1,51 & 0.58 \\
Right relative PFL volume $^{\mathrm{c}}$ & 0.002 & - & 1.28 & 1,61 & 0.26 \\
Left relative PFL volume $^{\mathrm{c}}$ & 0.005 & - & 2.89 & 1,61 & 0.094 \\
\hline
\end{tabular}

GI gyrification index, $O C D$ obsessive-compulsive disorder, $P F L$ prefrontal lobe, $F F$-statistic, $d f$ degrees of freedom, $p$ probability

a Analysis of covariance (ANCOVA; factor diagnosis, intervening variables: age, duration of education)

b Only right-handed subjects; analysis of variance (ANOVA; diagnosis, no intervening variables)

c Analysis of covariance (ANCOVA; factor diagnosis, intervening variable: age)

calculated the partial correlations between the obsession and compulsion subscore of the Y-BOCS and the GI (left and right). We found no significant relationship $(p>0.2)$. To take a closer look onto a possible correlation between severity of OCD and GI, we analysed if there is a difference in GI between OCD patients displaying only moderate obsessions-compulsions and OCD patients with severe symptoms (based on Y-BOCS rating). We found no significant differences between the subgroups (for left GI $p=0.98$; for right GI $p=0.53$ ). Nevertheless, building these two subgroups, we observed a significant difference in left GI only when comparing OCD patients with severe symptoms $(n=9)$ with controls (GI reduction 3.5\%; $F=3.02, d f=2,51 ; p=0.036$, ANCOVA), not when comparing OCD patients displaying only moderate symptoms $(n=11)$ with healthy controls.

Across groups we found no significant correlations between test performance and A-GI. However, in the subgroup of OCD patients we observed a significant correlation between the TOL total score and right GI $(\rho=0.61, p=0.046)$. This partial correlation did not survive correction for multiple testing.

\section{Prefrontal lobe volumes}

There was only a slight reduction at a trend level in left relative prefrontal lobe in OCD patients compared with healthy control subjects, but no significant differences in relative prefrontal lobe volumes between the diagnostic groups (left: $d f=1,61, F=2.89, p=0.094$; right: $d f=1,61, F=2.89, p=0.26$ ) (see Table 2).

Clinical variables (age of onset, duration of illness, BDI, Y-BOCS) did not show a significant correlation with prefrontal lobe volume in OCD patients. However, we could demonstrate a significant positive correlation between prefrontal A-GI and corresponding relative prefrontal lobe volume in healthy controls (right: $\mathrm{PC}=0.41 ; d f=35$; $p=0.01$; left: $\mathrm{PC}=0.40 ; d f=35 ; p<0.005)$, but not in OCD patients (right: $\mathrm{PC}=0.17 ; p=$ n.s.; left: $\mathrm{PC}=0.30$; $p=$ n.s.).

\section{Influence of medication}

Exact medication records were available for $20 \mathrm{OCD}$ patients: eight receiving SSRIs, three receiving tricyclic antidepressants (TCA), and nine patients receiving no antidepressants. To rule out the influence of medication, we repeated our analysis including only the nine medicationnaïve OCD patients. We found that this subgroup of unmedicated OCD patients displayed significant reduced prefrontal GI on both hemispheres compared with control subjects (GI right 3.7\% reduction in OCD patients; 2.113 vs. $2.195 ; p=0.003$ and GI left $3.4 \%$ reduction in OCD patients; 2.056 vs. $2.129 ; p=.004)$. This underlines that medication could not be responsible for the GI difference between OCD patients and control subjects.

\section{Discussion}

In our study, OCD patients demonstrated significant leftsided hypogyrification and at a trend level right-sided reduced gyrification of prefrontal lobe compared with healthy control subjects. This difference occurred in the absence of significant prefrontal lobe volume differences. Interestingly, we found a significant correlation between relative prefrontal lobe volumes and gyrification (A-GI) in control subjects, but not in OCD patients. This may reflect that reduced cortical folding in the prefrontal lobe serves as an independent biological vulnerability marker for OCD. A not-significant association between volume and folding in OCD patients could strongly point towards disturbed neurodevelopment or brain maturation in OCD patients. Volumetric and functional abnormalities of prefrontal and temporo-limbic cortical regions in OCD were found in several imaging studies, but this is the first study to report on gyrification and cortical folding in OCD patients. In so far these results have to be replicated in further investigations with different samples to prove this finding as a stable marker of disturbances in late neurodevelopment in OCD patients. Interestingly, a recent volumetric MRI study showed that thalamic volumes were significantly negatively correlated with orbitofrontal cortex (OFC) volumes in OCD patients, but not in healthy subjects [35]. The authors suggested with additional support of a meta analysis that structural alterations of both the thalamus and the OFC are inversely and specifically related in OCD. This fits 
to the results of our study that not only volume alterations of one brain area but also the relationship between connecting brain regions (e.g. thalamus and OFC) or mismatches between structural parameters (e.g. prefrontal lobe volume and GI) may contribute to the neurobiological vulnerability for OCD.

While the meaning of GI is not completely understood, it seems to be obvious that cortical folding serves as a marker of late ontogenetic brain development. Axonal tracts begin to develop before the onset of cortical folding [32] and lesions of tracts effect different gyral patterns [31] suggesting the importance of axonal connectivity to cortical folding. This assumption is in line with the tension-based theory of morphogenesis [42]. According to this model, differences in mechanical tension along axons connecting different cortical regions effect cortical folding. The gyrification pattern specific to humans is largely complete by birth and displays smooth and continuous development. Gyri and sulci appear in a specific order: the least variable "primary" gyri are first to appear, followed by the moderately variable "secondary" gyri, and then finally, the most variable "tertiary" gyri develop [2]. In humans, the sulco-gyral development continues until early adulthood. Although maximal GI values are reached in infancy, a process of "compensation" allows for continued growth to be accommodated by compensatory tertiary folding which maintains GI levels [2].

However, focal hypo- or hyper-gyria can be a consequence of disturbed fetal blood supply, intrauterine infection, or prenatal toxic events in human subjects as well. Animal experiments have demonstrated wide ranging misconnections in the neighborhood of focal disturbances of gyrification [9]. Disturbed gyrification, as now detected in OCD and as previously described in schizophrenia, seems to be associated with an abnormal development of cortical connectivity.

Structural MRI investigations revealed evidence for a reduction of orbitofrontal volume in OCD patients compared with healthy controls, strongly indicating the involvement of the prefrontal cortex in the pathogenesis of OCD [19]. In our study, OCD patients demonstrated tendentially but not significantly smaller relative prefrontal lobe volumes on the left side compared with healthy controls; nevertheless, prefrontal A-GI differed significantly on the left side and at a trend level on the right side. Interestingly, we found a significant correlation between relative prefrontal lobe volumes and A-GI only in control subjects, but not in OCD patients. This implies the possible implications discussed earlier. It could mean that reduced cortical folding in the prefrontal lobe is a biological vulnerability marker independent from reduced prefrontal lobe volume in OCD patients. Alternatively or additionally, the not-significant association of volume and folding in OCD patients could support strongly the hypothesis of a disturbed neurodevelopment or brain maturation in OCD.

Functional imaging studies [23] demonstrate abnormalities of glucose metabolism in the prefrontal cortex in OCD patients, which normalize after the successful treatment of symptoms [18]. This finding is another hint of the clinical relevance of the prefrontal cortex for the pathogenesis of OCD.

In one recent study, relatives of OCD cases from the community had a 8.5 -fold increase in the prevalence of definite OCD, and relatives from OCD cases from outpatient clinics had a 4.9-fold increase in the prevalence of definite OCD compared with relatives from control cases [11]. Older twin studies on OCD found concordance rates in monozygotic versus dizygotic twins of 80 versus $20 \%$ [17] and 87 versus $47 \%$ [7], altogether suggesting that there is a high genetic vulnerability. A family study investigating GI in affected and unaffected first degree relatives of schizophrenia patients showed focal hypergyrification also in unaffected family members supporting a genetic liability of GI [8].

Our findings of a significant negative correlation between prefrontal GI and age differ from the results of other investigations showing that the GI is age-independent [2], especially when we found the correlation in both, OCD patients and healthy control subjects. It has been previously reported that GI is a stable measure, fixed in early development and therefore relatively independent of age [48]. It is theoretically possible that medications taken by OCD patients may have affected these results, but then the effect of drugs would not be seen in healthy controls, as it could be demonstrated in our study. In addition, other studies have also identified age-related changes in GI $[6,36]$. This suggests that GI may not be as stable a measure as previously thought to be and raises the possibility that, while the degree of cortical folding may relate to early developmental factors, GI itself is vulnerable to other factors such as age-related changes in the shape of gyri and the depth of sulci [22]. It was reported that sulcal depth reduces with age, making the curvature at the crown of the gyri more gradual [25]. These effects give rise to a shortening of the inner contour and hence lower GI values. In turn, this raises the possibility that the differences in GI which we report may result from a process occurring after the early developmental period. Furthermore, GI did correlate significantly with education in the control subjects, a result that could not be found in previous studies of our research group. In this sample, we did not repeat the measurement with A-GI by manual tracing and calculating the manual measured GI (M-GI) used in the schizophrenia studies, but we compared both methods in a sample of twins. We found 
that although measured A-GI is not numerically identical with the M-GI (A-GI is lower), the observed differences between the diagnostic groups were similar [46].

There is an increased prevalence of comorbid OCD in patients with schizophrenia, reaching from 0.5 to $59.2 \%$, and of obsessive-compulsive symptoms (OCS) in schizophrenia from 3.5 to $46 \%$, depending on the specific sample [29]. There is a controversial discussion going on if this increased prevalence reflects a true comorbidity or a diagnostic subcategory of schizophrenia. There are some hints that schizophrenic patients with OCS showed a different extent of volume reductions compared with schizophrenic patients without OCS. In this subgroup, the volume reductions were more pronounced in, e.g., left hippocampus and frontal lobes [1]. In addition, in some studies schizophrenic patients with OCS demonstrated more neurological soft signs, motor symptoms including catatonia, and extrapyramidal symptoms [29]. Until today, there are no studies available measuring GI in schizophrenia patients with OCS. Nevertheless, it can be speculated that these patients may show a more pronounced reduced GI compared with schizophrenia patients without OCS.

Some limitations of this study should be borne in mind when interpreting the results. First, although the number of subjects is still relatively large in comparison to other imaging studies, the results are still in need of replication. Second, we did not find a correlation of reduced gyrification in OCD patients to clinical variables; therefore, the clinical impact of our finding beyond that of increased vulnerability for the development of OCD still remains unclear. Third, we did not assess depressive (BDI) and OCD (Y-BOCS) symptoms in the healthy control group for analysing a possible relationship between minor degree symptomatology and GI in healthy people. At least, we did not correct for multiple testing, which limits our results. In our small sample, we focused primarily on our hypothesis that GI is different between OCD patients and controls. All other analyses were secondary analyses, not causally linked to our primary outcome. Adjustment for multiple testing would lead to nonsignificant results in nearly all of our primary and secondary outcome parameters.

While structural MRI studies revealed evidence for brain abnormalities in OCD, gyrification has not been studied so far in OCD patients. Here, for the first time, we have found evidence of reduced prefrontal gyrification in OCD. Our findings support and extend other studies reporting structural and functional brain abnormalities of prefrontal cortex in OCD and provide further evidence that OCD is associated with neurodevelopmental processes affecting prefrontal cortex and its associated executive functions.
Acknowledgments T. Wobrock is a member of a speakers' bureau for Alpine Biomed, AstraZeneca, Eli Lilly, Essex and Janssen-Cilag, and has accepted paid speaking engagements in industry-sponsored symposia from AstraZeneca, Bristol-Myers-Squibb, Eli-Lilly, Janssen Cilag, Novartis and Pfizer, and travel or hospitality not related to a speaking engagement from Astra Zeneca, Bristol-Myers-Squibb, Eli Lilly, Janssen Cilag, and Sanofi-Synthelabo, and received a research grant from Astra Zeneca. P. Falkai is a member of a speakers' bureau for AstraZeneca, Eli Lilly, Janssen-Cilag and Lundbeck, and has accepted paid speaking engagements in industry-sponsored symposia from AstraZeneca, Bristol-Myers-Squibb, Eli-Lilly, Janssen-Cilag, Lundbeck and Pfizer, and travel or hospitality not related to a speaking engagement from Astra Zeneca, Bristol-Myers-Squibb, Eli Lilly, Janssen Cilag, Lundbeck and Sanofi-Synthelabo, and received a research grant from Astra Zeneca. H. Scherk is a member of a speakers' bureau for AstaZeneca and Eli Lilly and has accepted paid speaking engagements in industry-sponsored symposia from AstraZeneca and Eli-Lilly and travel or hospitality not related to a speaking engagement from Eli Lilly. A. McIntosh is supported by the Health Foundation UK and has a research grant from the Wyeth/Translational Medical Research Institute in Scotland. S. M. Lawrie is supported by the Sackler Foundation and has a research grant from the Wyeth/Translational Medical Research Institute in Scotland. T. W. Moorhead is supported by the Medical Research Council UK. O. Gruber, S. Kraft, A. Klinghardt, W. Reith and T. Schneider-Axmann report no competing interests.

\section{Conflict of interest statement None.}

Open Access This article is distributed under the terms of the Creative Commons Attribution Noncommercial License which permits any noncommercial use, distribution, and reproduction in any medium, provided the original author(s) and source are credited.

\section{References}

1. Aoyama F, Iida J, Inoue M, Iwasaka H, Sakiyama S, Hata K, Kishimoto T (2000) Brain imaging in childhood- and adolescence-onset schizophrenia associated with obsessive-compulsive symptoms. Acta Psychiatr Scand 102(1):32-37

2. Armstrong E, Schleicher A, Omran H, Curtis M, Zilles K (1995) The ontogeny of human gyrification. Cereb Cortex 5:56-63

3. Ashburner J, Friston KJ (2000) Voxel-based morphometry-the methods. Neuroimage 11:805-821

4. Bebbington PE (1998) Epidemiology of obsessive-compulsive disorder. Br J Psychiatry 35(35):2-6

5. Beck AT, Ward CH, Mendelson M, Mock J, Erbaugh J (1961) An inventory for measuring depression. Arch Gen Psychiatry 4:561-571

6. Bonnici HM, Moorhead TW, Stanfield AC, Harris JM, Owens DG, Johnstone EC, Lawrie SM (2007) Pre-frontal lobe gyrification index in schizophrenia, mental retardation and comorbid groups: an automated study. Neuroimage 35(2):648-654

7. Carey G, Gottesmann II (1981) Twin and family studies of anxiety, phobic, and obsessive disorders. In: Klein DF, Rabkin JG (eds) Anxiety: new research and changing concepts. Raven Press, New York, pp 117-136

8. Falkai P, Honer WG, Kamer T, Dustert S, Vogeley K, SchneiderAxmann T, Dani I, Wagner M, Rietschel M, Müller DJ, Schulze TG, Gaebel W, Cordes J, Schönell H, Schild HH, Block W, Träber F, Steinmetz H, Maier W, Tepest R (2007) Disturbed frontal gyrification within families affected with schizophrenia. J Psychiatr Res 41(10):805-813

9. Goldman-Rakic PS (1980) Morphological consequences of prenatal injury in the primate brain. Prog Brain Res 53:3-19 
10. Goodman WK, Price LH (1992) Assessment of severity and change in obsessive compulsive disorder. Psychiatr Clin North Am 15(4):861-869

11. Grabe HJ, Ruhrmann S, Ettelt S, Buhtz F, Hochrein A, SchulzeRauschenbach S, Meyer K, Kraft S, Reck C, Pukrop R, Freyberger HJ, Klosterkötter J, Falkai P, John U, Maier W, Wagner M (2006) Familiarity of obsessive-compulsive disorder in nonclinical and clinical subjects. Am J Psychiatry 163(11):1986-1992

12. Ha TH, Yoon U, Lee KJ, Shin YW, Lee JM, Kim IY, Ha KS, Kim SI, Kwon JS (2005) Fractal dimension of cerebral cortical surface in schizophrenia and obsessive-compulsive disorder. Neurosci Lett 384(1-2):172-176

13. Harris JM, Yates S, Miller P, Best JJ, Johnstone EC, Lawrie SM (2004) Gyrification in first-episode schizophrenia: a morphometric study. Biol Psychiatry 55:141-147

14. Harris JM, Whalley H, Yates S, Miller P, Johnstone EC, Lawrie SM (2004) Abnormal cortical folding in high-risk individuals: a predictor of the development of schizophrenia? Biol Psychiatry 56(3):182-189

15. Heaton RK, Chelune GJ, Talley JL, Kay GG, Curtiss G (1993) Wisconsin card sorting test manual—revised and expanded. PAR, Florida

16. Henseler I, Gruber O, Kraft S, Krick C, Reith W, Falkai P (2008) Compensatory hyperactivations as markers of latent working memory dysfunctions in patients with obsessive-compulsive disorder. J Psychiatr Neurosci 33(3):209-215

17. Inouye E (1965) Similar and dissimilar manifestations of obsessive-compulsive neurosis in monozygotic twins. Am J Psychiatry 121:1171-1175

18. Kang DH, Kwon JS, Kim JJ, Youn T, Park HJ, Kim MS, Lee DS, Lee MC (2003) Brain glucose metabolic changes associated with neuropsychological improvements after 4 months of treatment in patients with obsessive-compulsive disorder. Acta Psychiatr Scand 107(4):291-297

19. Kang DH, Kim JJ, Choi JS, Kim YI, Kim CW, Youn T, Han MH, Chang KH, Kwon JS (2004) Volumetric investigation of the frontal-subcortical circuitry in patients with obsessive-compulsive disorder. J Neuropsychiatry Clin Neurosci 16(3):342-349

20. Karno M, Golding JM, Sorenson SB, Burnam A (1988) The epidemiology of obsessive-compulsive disorder in five US communities. Arch Gen Psychiatry 45:1094-1099

21. Knapp M, Henderson J, Patel A (2002) Costs of obsessivecompulsive disorder: a review. In: Maj M, Sartorious N, Okasha A, Zohar J (eds) Evidence and experience in psychiatry: obsessive-compulsive disorder, 2nd edn. WPA Series, Wiley, pp 253273

22. Kochunov P, Mangin JF, Coyle T, Lancaster J, Thompson P, Riviere D, Cointepas Y, Regis J, Schlosser A, Royall DR, Zilles K, Mazziotta J, Toga A, Fox PT (2005) Age-related morphology trends of cortical sulci. Hum Brain Mapp 26(3):210-220

23. Kwon JS, Kim JJ, Lee DW, Lee JS, Lee DS, Kim MS, Lyoo IK, Cho MJ, Lee MC (2003) Neural correlates of clinical symptoms and cognitive dysfunctions in obsessive-compulsive disorder. Psychiatry Res Neuroimaging 122:37-47

24. Lacerda AL, Dalgalarrondo P, Caetano D, Camargo EE, Etchebehere EC, Soares JC (2003) Elevated thalamic and prefrontal regional cerebral blood flow in obsessive-compulsive disorder: a SPECT study. Psychiatry Res 123:125-134

25. Magnotta VA, Andreasen NC, Schultz SK, Harris G, Cizadlo T, Heckel D, Nopoulos P, Flaum M (1999) Quantitative in vivo measurement of gyrification in the human brain: changes associated with aging. Cereb Cortex 9:151-160

26. Mataix-Cols D, Wooderson S, Lawrence N, Brammer MJ, Speckens A, Phillips ML (2004) Distinct neural correlates of washing, checking, and hoarding symptom dimensions in obsessive-compulsive disorder. Arch Gen Psychiatry 61:564-576
27. Moorhead TW, Harris JM, Stanfield AC, Job DE, Best JJ, Johnstone EC, Lawrie SM (2006) Automated computation of the gyrification index in prefrontal lobes: methods and comparison with manual implementation. Neuroimage 31:1560-1566

28. Oswald WD, Roth E (1987) Der Zahlen-Verbindungs-Test (ZVT): Handanweisung (2. Aufl.). Hogrefe, Göttingen

29. Patel DD, Laws KR, Padhi A, Farrow JM, Mukhopadhaya K, Krishnaiah R, Fineberg NA (2009) The neuropsychology of the schizo-obsessive subtype of schizophrenia: a new analysis. Psychol Med 12:1-13

30. Pujol J, Soriano-Mas C, Alonso P, Cardoner N, Mench $\tilde{A}^{3} \mathrm{n} J M$, Deus J, Vallejo J (2004) Mapping structural brain alterations in obsessive-compulsive disorder. Arch Gen Psychiatry 61(7):720730

31. Rakic P (1988) Specification of cerebral cortical areas. Science 241:170-176

32. Rakic P (2000) Setting the stage of cognition: Genesis of the primate cerebral cortex. In: Gazzinga MS (ed) The new cognitive neurosciences, 2nd edn. MIT Press, London

33. Rosenberg DR, Keshavan MS (1998) Toward a neurodevelopmental model of obsessive-compulsive disorder. Biol Psychiatry 43:623-640

34. Rotge JY, Guehl D, Dilharreguy B, Tignol J, Bioulac B, Allard M, Burbaud P, Aouizerate B (2009) Meta-analysis of brain volume changes in obsessive-compulsive disorder. Biol Psychiatry 65(1):75-83

35. Rotge JY, Dilharreguy B, Aouizerate B, Martin-Guehl C, Guehl D, Jaafari N, Langbour N, Bioulac B, Tignol J, Allard M, Burbaud P (2009) Prog Neuropsychopharmacol. Biol Psychiatry 33(4):682-687

36. Sallet PC, Elkis H, Alves TM, Oliveira JR, Sassi E, Campi De Castro C, Busatto GF, Gattaz WF (2003) Reduced cortical folding in schizophrenia: an MRI morphometric study. Am J Psychiatry $160(9)$ : $1606-1613$

37. Shallice T (1982) Specific impairments in planning. Philos Trans R Soc Lond B Biol Sci 298:199-209

38. Stein DJ, Goodman WK, Rauch SL (2000) The cognitiveaffective neuroscience of obsessive-compulsive disorder. Curr Psychiatry Rep 2:341-346

39. Stein DJ, Arya M, Pietrini P, Rapoport JL, Swedo SE (2006) Neurocircuitry of disgust and anxiety in obsessive-compulsive disorder: a positron emission tomography study. Metab Brain Dis 21:267-277

40. Valente AA, Miguel EC, Castro CC, Amaro E, Duran FL, Buchpiguel CA, Chitnis X, McGuire PK, Busatto GF (2005) Regional gray matter abnormalities in obsessive-compulsive disorder: a voxel-based morphometry study. Biol Psychiatry 58(6):479-487

41. Van den Heuvel OA, Veltman DJ, Groenewegen HJ, Cath DC, van Balkom AJ, van Hartskamp J, Barkhof F, van Dyck R (2005) Frontal-striatal dysfunction during planning in obsessive-compulsive disorder. Arch Gen Psychiatry 62:301-310

42. Van Essen DC (1997) A tension-based theory of morphogenesis and compact wiring in the central nervous system. Nature 385:313-318

43. Vogeley K, Schneider-Axmann T, Pfeiffer U, Tepest R, Bayer TA, Bogerts B, Honer WG, Falkai P (2000) Disturbed gyrification of the prefrontal region in male schizophrenic patients: a morphometric postmortem study. Am J Psychiatry 157(1):34-39

44. Vogeley K, Tepest R, Pfeiffer U, Schneider-Axmann T, Maier W, Honer WG, Falkai P (2001) Right frontal hypergyria differentiation in affected and unaffected siblings from families multiply affected with schizophrenia: a morphometric MRI study. Am J Psychiatry 158(3):494-496

45. Weinberger DR (1995) From neuropathology to neurodevelopment. Lancet 346:552-557 
46. Wobrock T, McIntosh AM, Gruber O, Droese U, Tepest R, Moorhead TW, Schneider-Axmann T, Falkai P (2008) Heretability of prefrontal cortex gyrification: a twin study. Eur Arch Psychiatry Clin Neurosci 258(4):17

47. Wobrock T, Sittinger H, Behrendt B, D'Amelio R, Falkai P (2009) Comorbid substance abuse and brain morphology in recent-onset psychosis. Eur Arch Psychiatry Clin Neurosci 259(1):28-36

48. Zilles K, Armstrong E, Schleicher A, Kretschmann HJ (1988) The human pattern of gyrification in the cerebral cortex. Anat Embryol (Berl) 179:173-179 